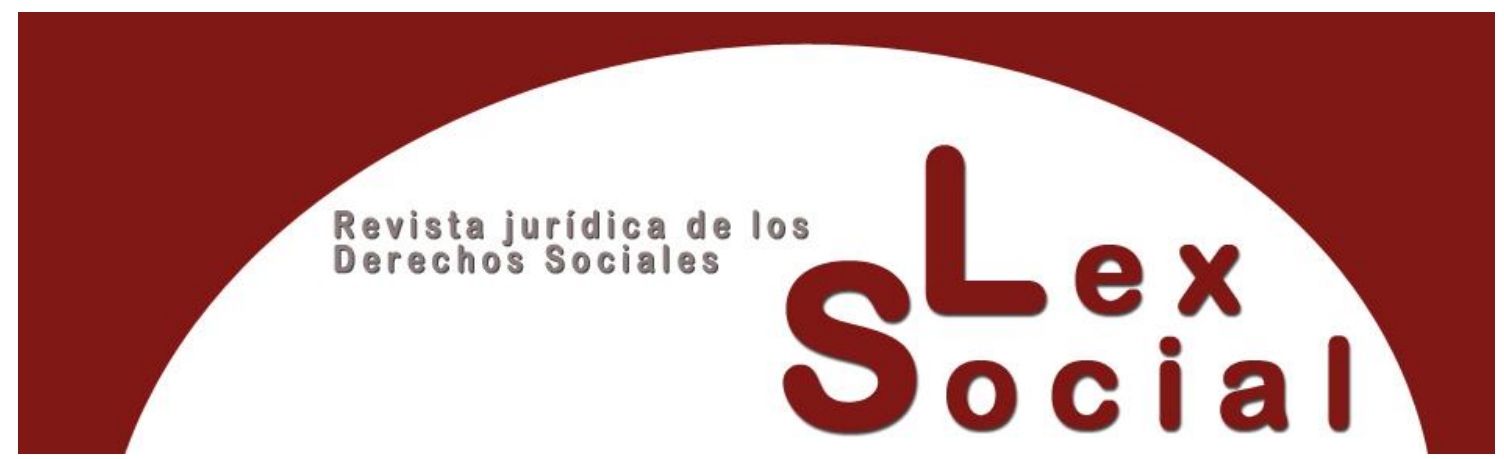

\title{
IN DUBIO PRO MULIERE. HIJAS CUIDADORAS Y PENSIÓN A FAVOR DE FAMILIARES. INTERPRETACIÓN FLEXIBLE Y CON PERSPECTIVA DE GÉNERO DEL REQUISITO DE CONVIVENCIA: A propósito de la STSJ de Canarias de 13 de marzo de 2020 (Rec. 1400/2019) ${ }^{1}$
}

\section{IN DUBIO PRO MULIERE. CAREGIVING DAUGHTERS AND PENSION FOR FAMILY MEMBERS. FLEXIBLE INTERPRETATION (AND WITH A GENDER PERSPECTIVE) OF THE COHABITATION REQUIREMENT: Regarding the Sentence of the Social Chamber of the High Court of Justice of Canary Islands of March 13, 2020 -Recourse number 1400/2019-}

Cómo citar este trabajo: Martínez Barroso, M. R. (2021). In dubio pro muliere. Hijas cuidadoras y pensión en favor de familiares. Interpretación flexible y con perspectiva de género del requisito de convivencia: A propósito de la STSJ de Canarias de 13 de marzo de 2020 (Rec. 1400/2019). Lex Social, Revista De Derechos Sociales, 11 (2), 842-866. https://doi.org/10.46661/lexsocial.5971

\section{RESUMEN}

Bajo la genérica denominación de prestaciones de muerte y supervivencia contempla el ordenamiento la protección (además de la específicamente dispensada al cónyuge viudo, al superviviente de una pareja de hecho y a los huérfanos) de determinados familiares del causante en los que concurran cumulativamente ciertos requisitos en el momento del hecho causante: convivir con el causante y a sus expensas, al menos durante los dos años anteriores; carecer de medios de

\footnotetext{
${ }^{1}$ Trabajo realizado al amparo del proyecto de investigación: "La prestación de servicios socio-sanitarios en el ámbito rural de Castilla y León: apostando por un bienestar integral”, financiado por la Junta de Castilla y León.
}

\section{(cc) BY-NC-SA}


subsistencia propios, cifrados en el Salario Mínimo Interprofesional (SMI); no tener derecho a otra pensión pública o prestación periódica de la Seguridad Social e inexistencia de familiares con obligación y posibilidad de prestarles alimentos (recayendo esta obligación, según lo establecido en los artículos 142 y 143 del Código Civil, en los cónyuges, ascendientes y descendientes).

Entre los posibles beneficiarios de tal protección, en concreto, de la pensión a favor de familiares, contempla el ordenamiento, entre otros, a los hijos y hermanos (varones y mujeres) de beneficiarios de pensiones de incapacidad permanente o de jubilación contributivas, mayores de 45 años y solteros, separados judicialmente, divorciados o viudos, que acrediten dedicación prolongada al cuidado del causante.

Y precisamente a propósito del alcance que deba otorgarse a tales cuidados versa la sentencia objeto de comentario, dictada por el Tribunal Superior de Justicia de Canarias el pasado 13 de marzo de 2020, en la cual se profundiza en el análisis del concepto de convivencia (se daba la circunstancia en el caso de que la actora se encontraba empadronada, junto con su hijo de 19 años de edad, en la misma calle y número que su progenitora, pero en pisos diferentes), llegando a la conclusión de que la convivencia exigida en las prestaciones a favor de familiares debe interpretarse más allá de la estricta cohabitación física, pues una interpretación excesivamente formalista del concepto dejaría fuera de protección situaciones reales de necesidad y dependencia, padecidas mayoritariamente por mujeres. En el asunto comentado, en la medida en que la hija ha venido cuidando y atendiendo las necesidades vitales de su madre mientras vivía y lo requería, con una asistencia continua (día y noche) por vivir en el mismo edificio, debe priorizarse en la valoración de este requisito que tiene un componente claramente social, humano y asistencial que quedaría diluido de hacerse una interpretación mecanicista y puramente física. Así, pese a la ausencia de esa cohabitación física se procede a reconocer la pensión a la hija de la pensionista, interpretando el artículo 226.2 TRLGSS a la luz de los principios de igualdad y no discriminación por razón de sexo. La aplicación de dicho canon hermenéutico permite al Tribunal resolver la controvertida cuestión desde una perspectiva de género que obliga a una contextualización y actuación conforme al principio pro persona y en favor de una mayor protección de los derechos humanos.

Palabras Clave: pensión a favor de familiares, hijas cuidadoras, convivencia, perspectiva de género, interpretación flexible.

\section{SUMMARY}

Under the generic name of death and survivors benefits, social security legislation provides protection for certain relatives of the dead person (in addition to that specifically granted to the surviving spouse/partner and orphans). Members of the 
deceased's family have to cumulatively fulfil certain requirements at the time of the causal event: they must have lived with and depended financially on the deceased for at least two years prior to their death; they must lack their own means of subsistence, equivalent to the Minimum Interprofessional Wage (SMI); they mustn't be entitled to any other periodical pension or temporary allowance of Social Security and, finally, they must not have relatives who are obliged to provide adequate maintenance to them (this duty falls on spouses, ascendants and descendants, according to Articles 142 and 143 of Spanish Civil Code).

The social security legislation includes among the possible beneficiaries of this pension for family members, among others, children and siblings (male and female) of beneficiaries of permanent disability or contributory retirement pensions, persons over 45 years of age and single, judicially separated, divorced or widowed persons, who can prove that they have devoted a long period of time caring for the deceased.

The judgment in question focuses precisely on the scope of the family caregiving, analysing, in particular, the concept of cohabitation (in a case where the relative was registered, together with her 19-year-old son, in the same street and number as his mother, but in different flats). The High Court of Justice of Canary Islands concludes that the cohabitation required to qualify for the pension for family members must be interpreted beyond strict physical cohabitation, because an excessively formalistic interpretation of the concept would exclude from protection real situations of need and dependency, suffered mostly by women. In this particular case, the daughter has been caring for and attending to the vital needs of her mother, with continuous assistance (day and night), and this is prioritised in the assessment of this requirement, which has a clearly social, human and assistance component (only in a mechanistic and purely physical interpretation would it be become blurred).

Consequently, despite the absence of such physical cohabitation, the Court decided to recognise the pension to the daughter of the deceased, interpreting article 226.2 of the Spanish Social Security Act, in the light of the principles of equality and nondiscrimination on grounds of sex. The application of this hermeneutic canon allows the Court to resolve the controversial issue from a gender perspective which makes it necessary to consider a contextualisation and action in line with the pro personae principle and in favour of greater protection of human rights.

Keywords: pension for family members, caregiving daughters, cohabitation, gender perspective, flexible interpretation. 
SUMARIO

\section{Cuestión litigiosa}

II. Recordatorio de la normativa aplicable

III. La aplicación de la perspectiva de género en derechos de protección social

$I V$. El rol femenino de los cuidados y su cobertura a través de la suscripción de convenios con la Seguridad Social

$V$. Interpretación flexible del concepto de convivencia en las pensiones a favor de familiares

VI. Interpretación del requisito de convivencia con perspectiva de género

VII. Conclusión final

Bibliografía

Anexo

\section{Cuestión litigiosa}

Se reclama por la actora el acceso a las prestaciones en favor de familiares, reguladas en el artículo 226 del Real Decreto Legislativo 8/2015, de 30 de octubre, por el que se aprueba el texto Refundido de la Ley General de la Seguridad Social (TRLGSS), dentro del Capítulo XIV, correspondiente a las prestaciones por muerte y supervivencia. Por parte del INSS se denegó a la demandante (de estado civil, divorciada) el derecho al cobro de estas prestaciones "por no haber convivido con el causante y a su cargo". La sentencia de la instancia desestimó la demanda planteada por la actora reiterando el criterio del INSS, al considerar que, aun viviendo en la misma calle y número de edificio que su madre y causante, no obstante, lo hacían en pisos diferentes, por lo cual, tal y como literalmente se recoge en el fundamento de derecho único de la sentencia, "No puede deducirse de estos hechos que la actora viviera por cuenta de su madre y conviviera con ella por más que habitara en el piso de arriba y le prestase atención y cuidados en el piso de abajo, lo que nadie discute. No se ha realizado prueba alguna que explique que las viviendas en realidad eran una sola, argumento que no ha sido utilizado en el acto del juicio, por más que figurase en la demanda. Por lo tanto, no se acredita convivencia en el mismo domicilio, sino solo cercanía, por lo que ha de desestimarse la demanda (...)".

El fallo de la Sentencia de instancia ${ }^{2}$ desestima la pretensión promovida por la actora frente al INSS y TGSS, absolviendo a la entidad demandada de las pretensiones deducidas.

\footnotetext{
${ }^{2}$ Sentencia del Juzgado de lo Social núm. 5 de Las Palmas de Gran Canaria, de 3 de octubre de 2019, en autos promovidos sobre reclamación de prestaciones a favor de familiares, que es revocada.
} 
En el recurso de suplicación, como primer y segundo motivo del recurso, la recurrente solicita la revisión de los hechos declarados probados a la vista de las pruebas documentales y periciales practicadas, subrayando que sí ha convivido con su madre pensionista, frente a lo cual el INSS considera (defectos formales aparte, al no hacer propuesta alternativa a los hechos probados que pretende cambiar) que debe estarse a los datos de empadronamiento que constan, cuestionando la "objetividad" de las declaraciones testificales efectuadas por la amiga y hermana de la actora (a la sazón, las personas que tenían conocimiento directo de los hechos que se pretendían probar; esto es, la relación de la actora con su madre, el tiempo convivido con ella en vida y los cuidados que le brindó). En todo caso, la Sala desestima las revisiones fácticas planteadas, al no hacerse propuesta alternativa de revisión, pues ello contraviene los requisitos establecidos en el artículo 193 b) en relación con el artículo 196.3 de la Ley Reguladora de la Jurisdicción Social (LRJS).

En el motivo tercero y cuarto del recurso, se cuestiona la fundamentación jurídica de la sentencia que lleva a la magistrada de instancia a la convicción de que no se ha probado en el caso que nos ocupa el requisito de la convivencia exigido por el artículo 226 TRLGSS para que la actora pueda tener acceso a la prestación reclamada.

La recurrente no señala formalmente la infracción de normas sustantivas, ni tampoco que estos motivos se plantean, con amparo en el artículo 193 c) LRJS, pero resulta implícito de la literalidad de estos dos motivos que lo que se cuestiona es la infracción del artículo 226.2 TRLGSS, al entender la recurrente que contrariamente a lo que entendió la magistrada de la instancia, sí convivía con su madre pensionista, hasta que esta falleció.

En relación a estos dos motivos, por lo que respecta a los defectos formales sin transcendencia o que se subsanan a través del desarrollo del propio motivo del recurso, la Sala otorga prevalencia al principio "pro actione", pues lo contrario podría suponer una vulneración de un derecho fundamental (artículo $24 \mathrm{CE}$ ), debiéndose evitar en estos casos una interpretación excesivamente rigorista ${ }^{3}$. El principio "pro actione" opera en este caso sobre los requisitos establecidos legalmente para acceder al proceso, impidiendo que determinadas aplicaciones o interpretaciones relativas a ellos eliminen u obstaculicen injustificadamente el derecho a que un órgano judicial conozca y resuelva en Derecho sobre la pretensión a él sometida.

Este especial rigor en el momento de valorar, desde la perspectiva constitucional, las decisiones judiciales que imposibilitan el conocimiento procesal de la cuestión de fondo

\footnotetext{
${ }^{3}$ Tal y como ha venido entendiendo con nitidez el Tribunal Constitucional, entre otras, en su sentencia núm. 112, de 3 de junio de 1997 (RTC 1997, 112), en cuya fundamentación jurídica se recoge expresamente que "Constituye un criterio reiteradamente mantenido por la jurisprudencia constitucional el de que el derecho a la tutela judicial efectiva se satisface también con una respuesta de inadmisión, si bien esta ha de estar fundada en una causa legal apreciada razonablemente por el órgano judicial”. Doctrina con la que el Tribunal Constitucional ha controlado las respuestas de inadmisión que determinan el cierre del acceso al proceso [Entre otras, SSTC 87/1986 (RTC 1986, 87); 216/1987 (RTC 1987, 216); 154/1992 (RTC 1992, 154); 55/1995 (RTC 1995, 55)], ya que la tutela judicial efectiva conlleva, como primera y esencial característica, el derecho de libre acceso de los ciudadanos a los Juzgados y Tribunales.
} 
ha sido aplicado, específicamente, en relación a algunas irregularidades formales de las demandas, que determinaron, en su día, su archivo. Supuestos estos sobre los que, además, se proyecta otro consolidado criterio mantenido por el propio Tribunal Constitucional, que, con carácter general, ha rechazado que los requisitos formales tengan sustantividad propia y resaltado que constituyen medios orientados a conseguir ciertas finalidades en el proceso, de modo que sus eventuales anomalías no pueden ser convertidas sin más en meros obstáculos formales impeditivos de la continuación de aquel, sino que resulta obligada una interpretación de tales defectos guiada por un criterio de proporcionalidad entre la finalidad que cumple la exigencia formal y la entidad real del defecto observado en ella, atendiendo a las consecuencias que se siguen para la efectividad del derecho a la tutela judicial ${ }^{4}$.

\section{Recordatorio de la normativa aplicable}

El carácter sumamente pedagógico de la sentencia comentada se refleja de manera fiel en el exhaustivo repaso realizado de la normativa, tanto legal como reglamentaria, reguladora de las prestaciones a favor de familiares, a partir del marco normativo configurado por el artículo 226 TRLGSS, que literalmente establece lo siguiente:

"En las normas de desarrollo de esta ley se determinarán aquellos otros familiares o asimilados que, reuniendo las condiciones que para cada uno de ellos se establezcan y previa prueba de su dependencia económica del causante, tendrán derecho a pensión o subsidio por muerte de este, en la cuantía que respectivamente se fije".

Centrando la atención en la modalidad contenida en el párrafo segundo del artículo 226 TRLGSS, que es la cuestionada en el caso debatido, los requisitos para acceder a esta prestación contributiva derivada de muerte y supervivencia son los siguientes:

$1^{\circ}$.- Ser hijos/as o hermanos/as de las personas beneficiarias de pensiones contributivas de jubilación o incapacidad permanente.

$2^{\circ}$.- Ser mayor de cuarenta y cinco años y soltera/o, divorciada/o viuda/o.

$3^{\circ}$.- Haber convivido con el causante y a su cargo, al menos con dos años de antelación al fallecimiento del causante

$4^{\mathrm{o}}$.- Acreditar dedicación prolongada al cuidado de la persona causante.

$5^{\circ}$.- Carecer de medios propios de vida.

En cuanto a la duración de los subsidios temporales hace, la ley se remite a las normas de desarrollo, considerando que quienes se encuentren en situación legal de separación "tendrán, respecto de sus ascendientes o descendientes, los mismos derechos que los que les corresponderían de estar disuelto su matrimonio".

\footnotetext{
${ }^{4}$ Entre otras muy reiteradas, SSTC 17/1985 (RTC 1985, 17); 49/1987 (RTC 1997, 49); 174/1988; 2/1989 (RTC 1989, 2) o 240/1991 (RTC 1991, 240).
} 
El Decreto 1646/1972, de 21 de junio dispone en su artículo 5 lo siguiente en relación a las hijas o hermanas de pensionistas de jubilación o invalidez: "Tendrán derecho a pensión los hijos o hermanos de pensionistas de jubilación o incapacidad permanente que, al tiempo del fallecimiento del causante, sean mayores de cuarenta y cinco años de edad y solteros o viudos, siempre que reúnan las condiciones exigidas [...] y acrediten dedicación prolongada al servicio del causante".

El Reglamento General, aprobado por Decreto 3158/1966, de 23 de diciembre, cuando determina la cuantía de las prestaciones económicas del Régimen General de la Seguridad Social y condiciones para el derecho a las mismas, preceptúa en su artículo 40, en cuanto a los beneficiarios de la pensión a favor de familiares, lo siguiente: “c) Que convivieran con el causante y a sus expensas, al menos con dos años de antelación al fallecimiento de aquel o desde la muerte del familiar con el que convivieran, si esta hubiere ocurrido dentro de dicho periodo; d) Que no tengan derecho a pensión del Estado, provincia o Municipio o a prestaciones periódicas de la Seguridad Social; e) Que, a juicio del órgano de gobierno competente, carezcan de medios de subsistencia y no queden familiares con obligación y posibilidades de prestarles alimentos, según la legislación civil”.

También la Orden Ministerial de 13 febrero 1967, por la que se establecen normas para la aplicación y desarrollo de las prestaciones de muerte y supervivencia del Régimen General de la Seguridad Social, enumera, en su artículo 22, quiénes son los beneficiarios de la pensión a favor de familiares, contemplando tres grupos de familiares y sus requisitos: 1) Nietos y hermanos del causante. 2) Madre y abuelas. 3) Padre y abuelos.

\section{La aplicación de la perspectiva de género en derechos de protección social}

Como es sabido, la transversalidad del principio de igualdad permite una interpretación y aplicación de las normas con perspectiva de género. En la controversia jurídica suscitada en el pronunciamiento comentado, el objeto de debate es el acceso a las denominadas prestaciones a favor de familiares, las cuales, a tenor de los datos estadísticos del INSS ${ }^{5}$, son percibidas mayoritariamente por las mujeres, en lógica correspondencia con el trabajo de los cuidados, que también recae mayoritariamente sobre ellas.

La invisibilidad de tareas que resultan imprescindibles para el desarrollo de la economía productiva, pero que, sin embargo, padecen una falta de reconocimiento, tales como la atención a las labores del hogar, los cuidados de personas dependientes (hijos e hijas y mayores) u otras responsabilidades familiares o personales con frecuencia provocan la expulsión femenina del mercado laboral o, al menos, limitan su participación, al estar fuertemente feminizadas, reforzando roles de género y estereotipos sociales difíciles de vencer ${ }^{6}$.

\footnotetext{
${ }^{5}$ Accesibles a través de la aplicación eSTADISS desde la página web: www.seg-social.es.

${ }^{6}$ Sobre el particular me he pronunciado en MARTÍNEZ BARROSO, $\mathrm{M}^{\mathrm{a}}$.R., "La protección social de las trabajadoras: la brecha de género en las prestaciones de jubilación y desempleo", en AA.VV. (RODRÍGUEZ ESCANCIANO, S. y MARTÍNEZ BARROSO, Mª.R., Dirs. y ÁLVAREZ CUESTA, H.,
} 
La consagración constitucional de la igualdad por razón de sexo y género como derecho fundamental y como principio normativo (artículo $14 \mathrm{CE}$ ) no impide que en la práctica sigan existiendo obstáculos y diferencias peyorativas contra las mujeres por la doble condición biológico-cultural sexo-género, que genera situaciones discriminatorias, en especial en el ámbito laboral y de la Seguridad Social. Por tanto, para combatirlas, el Poder Judicial se considera una pieza clave del sistema de garantías que permite asegurar la efectividad del derecho a la igualdad por razón de sexo, erradicando los comportamientos discriminatorios que persisten en el ámbito sociolaboral, integrando la metodología jurídica de juzgar con perspectiva de género ${ }^{7}$. Dicha justicia con perspectiva de género y su aplicación en la rama de lo Social no constituye una mera opción o recomendación para la buena práctica de la función jurisdiccional, sino que se erige en un auténtico mandato legal imperativo, que emana tanto de instrumentos internacionales suscritos por España como de la legislación nacional, erigiéndose en un parámetro resolutorio vinculante para el Poder Judicial en función de su sumisión al imperio de la ley (artículo $117 \mathrm{CE})^{8}$.

La justicia con perspectiva de género supone una especie de "transversalización (real) del principio de igualdad (gender mainstreaming), tal y como preceptúa el artículo 4 de la Ley 3/2007, de 22 de marzo, para la igualdad efectiva de mujeres y hombres, en relación con la previsión contenida en los artículos 9.1 y 14 de la Constitución, debiendo recordar el juego de los artículos 10 y 96 de la Carta Magna en la interpretación de los derechos fundamentales, de conformidad con los Tratados y Acuerdos internacionales ratificados por España" $"$. Por tanto, el principio de integración de la dimensión de género en la actividad judicial constituye una obligación normativizada y vinculante que se fundamenta en disposiciones internacionales, europeas y nacionales ${ }^{10}$. Es más, y en consonancia con lo dispuesto en el artículo 1.4 del Código Civil, el principio de igualdad de trato entre mujeres y hombres constituye una fuente del derecho con la eficacia propia de los principios informadores, erigiéndose además en un principio hermenéutico del derecho positivo ${ }^{11}$, que repercute directamente sobre los criterios de interpretación recogidos en el artículo 3.1 del Código Civil, al tratarse de un "método jurídico integrador

Coord.), La empleabilidad y la calidad en el empleo: apostando por la igualdad efectiva, Sepín, Madrid, 2019 , pp. 199 y ss.

${ }^{7}$ MOLINA NAVARRETE, C., La doctrina jurisprudencial por discriminación de género en el orden social, Wolters kluwer, Madrid, 2020, pp. 31 y 32.

${ }^{8}$ Sobre el particular, entre otros, LOUSADA AROCHENA, J.F., El derecho fundamental a la igualdad efectiva de mujeres y hombres, Tirant lo Blanch, Valencia, 2014 o "La integración de la perspectiva de género en la aplicación e interpretación de las normas por la jurisdicción social”, RDS, núm. 76, 2016, pp. 39 y ss.

${ }^{9}$ POYATOS MATAS, G., "Nueva sentencia con enfoque de género en el ejercicio del derecho a cuidar y trabajar: A propósito de la Sentencia de la Sala de lo Social del TSJ de Canarias de 27 agosto 2019 (rec. 533/2019)", IQual. Revista de Género e Igualdad, núm. 3, 2020, p. 152.

${ }^{10}$ RODRÍGUEZ GONZÁLEZ, S., "La perspectiva de género en la aplicación e interpretación de las normas por la Sala de lo Social del Tribunal Supremo", Trabajo y Derecho, núm. 76, 2021, p. 1.

11 JIMÉNEZ HIDALGO, A., "Juzgar con perspectiva de género en la jurisdicción de lo Social. ¿Es necesaria una reforma legislativa?", Revista de la Comisión de lo Social de Juezas y Jueces para la Democracia, núm. 197, 2019, p. 35. 
de la dimensión realista y evolutiva (cultural) del derecho en la necesaria dimensión normativista" 12 .

El interrogante que necesariamente surge a continuación es cuál sea el contenido concreto de esta metodología jurídica, a lo cual los expertos responden considerando que dicha metodología de análisis, equivalente a aplicar el derecho "con equidad de género", debe desplegarse "en aquellos casos en los que se involucren relaciones de poder asimétricas o patrones estereotípicos de género", exigiendo la integración del principio de igualdad en la interpretación y aplicación del ordenamiento jurídico, en la incesante búsqueda de soluciones equitativas ante situaciones desiguales de género"13.

En su fundamentación jurídica, la Sala aporta datos estadísticos del siguiente tenor: "a fecha 1 de diciembre de 2018, el número total de personas perceptoras de prestaciones a favor de familiares era de 42.281, de las que 29.360 eran mujeres; a fecha 1 de diciembre de 2019, el total ascendía a 43.156, de las que 29.596 eran mujeres. Ello se traduce en una abrumadora mayoría femenina que en porcentajes representa el 69,44\% en 2018 y el $68,57 \%$ en diciembre de 2019”.

Las cifras expuestas, que siguen la misma cadencia en 2020, revelan el incuestionable impacto de género que tienen las prestaciones analizadas y el lento avance hacia la igualdad (real), "lo que exige necesariamente extremar las cautelas judiciales en el abordaje de la controversia jurídica", que debe enfocarse de forma contextualizada "para evitar interpretaciones o impactos jurídicos que conlleven exclusiones, restricciones o distinciones dañinas, para los derechos humanos de las mujeres"14.

La aplicación de la perspectiva de género como método jurídico de análisis en el juicio de validez de las normas y prácticas implica, como con acierto ha señalado la propia doctrina científica, realizar una "interpretación de las leyes que garantice una mayor atención al contexto fáctico de desigualdad de roles y posiciones entre los sexos, como condición de efectividad de la igualdad jurídica" 15 . Por tanto, no cabe su aplicación en todos los casos en que esté implicada una mujer, sino solo en aquellos supuestos en los que se constate la existencia de una relación desequilibrada de poder, esto es, que se produzca un impacto de género como consecuencia de estereotipos o prejuicios sociales imperantes, de forma que se ha de identificar a la persona que se encuentra en situación de desigualdad por razón de género (sea una mujer o un hombre) ${ }^{16}$.

A tal fin, los órganos jurisdiccionales del orden social deben analizar la situación enjuiciada empleando el test de but for o de la sustitución (prueba de verificación

\footnotetext{
${ }^{12}$ MOLINA NAVARRETE, C., La doctrina jurisprudencial por discriminación de género en el orden social, op. cit., p. 57.

${ }^{13}$ POYATOS I MATAS, G., "Juzgar con perspectiva de género: una metodología vinculante de justicia equitativa", IQual. Revista de Género e Igualdad, núm. 2, 2019, pp. 7 y 8.

${ }^{14}$ Tal y como había establecido la propia STSJ de Canarias de 2 de julio de 2019 (Rec. 369/2019).

${ }^{15}$ MOLINA NAVARRETE, C., La doctrina jurisprudencial por discriminación de género en el orden social, cit., p. 52.

${ }^{16}$ AVILÉS PALACIOS, L., "Juzgar con perspectiva de género. Por qué y para qué", AMJE (Asociación de Mujeres Juezas de España), 29 de agosto de 2017.
} 
comparativa del resultado), para averiguar si las consecuencias jurídicas habrían sido las mismas o se habría producido un resultado contrario a la existencia de discriminación. Asimismo, deben asumir una "implicación proactiva" para depurar las discriminaciones existentes en el ordenamiento jurídico y en la realidad laboral y de Seguridad Social, promover la equidad real de oportunidades y beneficios en los casos en que surjan dudas interpretativas (in dubio pro muliere), integrar las lagunas jurídicas y corregir las antinomias (artículos 4 y 15 LOIEMH). Y todo ello con el necesario rigor en la aplicación de este método jurídico para evitar que con su labor (especialmente la "de suplencia o integración") se invada el espacio propio de la función legislativa ${ }^{17}$.

A tal fin, la Sala insular encuentra apoyo jurídico en la STS de 29 de enero de $2020^{18}$, dictada en Sala General, sobre acceso a prestaciones en favor de familiares derivadas de causante pensionista SOVI, recordando la importancia de los datos estadísticos como elemento objetivo para la detección de discriminaciones indirectas por razón de $\operatorname{sexo}^{19}$, y en la doctrina del propio Tribunal Constitucional, cuando indica que para abordar el análisis de la discriminación indirecta hay que ir "necesariamente a los datos revelados por la estadística" ${ }^{20}$. De ahí que una interpretación estricta y literal del precepto cuestionado pueda generar un impacto de género o una discriminación indirecta "al desplegar efectos desproporcionados sobre el colectivo femenino (...)”.

\section{El rol femenino de los cuidados y su cobertura a través de la suscripción de convenios con la Seguridad Social}

Como se anticipó, las prestaciones a favor de familiares (tanto pensiones como subsidios) son percibidas mayoritariamente por las mujeres, en lógica correspondencia con el trabajo de los cuidados, que también recae mayoritariamente sobre ellas. Y ello no es nada nuevo,

\footnotetext{
${ }^{17}$ MOLINA NAVARRETE, C., La doctrina jurisprudencial por discriminación de género en el orden social, cit., p. 48 y RODRÍGUEZ GONZÁLEZ, S., "La perspectiva de género en la aplicación e interpretación de las normas por la Sala de lo Social del Tribunal Supremo”, op. cit., p. 3.

${ }^{18}$ Rec. 3097/2017. En esta importante sentencia, el Tribunal Supremo lleva a cabo una reinterpretación desde un enfoque de género de la normativa aplicable en materia de Seguridad Social, en cuanto el principio de igualdad entre mujeres y hombres es un principio transversal que debe ser aplicado en las actuaciones de los poderes públicos, también del poder judicial, las cuales han de estar dirigidas hacia la consecución de una igualdad real y efectiva, evitando discriminaciones (directas o indirectas) por razón de género. Con este objetivo, el Tribunal Supremo aplica también por primera vez el concepto de "discriminación por asociación", construcción jurisprudencial del TJUE. Un interesante comentario a la misma en RIVAS VALLEJO, P., "Las beneficiarias de pensiones SOVI pueden causar prestaciones en favor de familiares", Revista de Jurisprudencia Laboral, núm. 2, 2020 o VILLAR CAÑADA, I.M., "La necesaria interpretación del sistema de Seguridad Social desde un enfoque de género. Comentario a la Sentencia del Tribunal Supremo (Sala de lo Social) núm. 79, de 29 de enero", Revista de Derecho de la Seguridad Social, núm. 26, 2021, pp. 133 y ss.

${ }^{19}$ La doctrina del TJUE ha consagrado el criterio de que la discriminación indirecta pueda ser demostrada por cualquier medio, incluidos los datos estadísticos, siempre que estos no se refieran a fenómenos meramente fortuitos o coyunturales y, además, resulten significativos. STJUE de 9 febrero 1999, SeymourSmith y Pérez, C-167/97 (TJCE 1999, 21) y, más recientemente, STJUE de 8 mayo 2019, Villar Laíz, C161/18 (TJCE 2019, 83); 3 octubre 2019, Schuch- Ghannadan, C-274/18 y ATJUE de 15 octubre 2019, AEAT C- 439/18 (TJCE 2019, 228) y C-472/18; entre otras.

${ }^{20}$ SSTC 128/1987 (RTC 1987, 128); 253/2004 (RTC 2004, 253) o 91/2019 (RTC 2019, 91).
} 
pues ya antes de la aprobación de la Ley 39/2006, de 14 de diciembre, de Promoción de la Autonomía Personal y Atención a las personas en situación de Dependencia (LAAD), las necesidades de las personas mayores y, de un modo más genérico, de las afectadas por situaciones de dependencia habían sido atendidas por las familias, con un soporte indudablemente femenino, apoyadas desde los sistemas de servicios sociales en los que estaban involucrados, prioritariamente, los ámbitos autonómicos y locales.

Con el ánimo de contextualizar la labor de cuidados al objeto de interpretar correctamente la normativa reguladora de las prestaciones a favor de familiares conviene recordar cómo con la ordenación legal de la dependencia, el Estado interviene de forma decisiva en este ámbito, creando el nuevo Sistema para la Autonomía y Atención a la Dependencia (SAAD) como instrumento esencial para el desarrollo y mejora del sistema de servicios sociales en España, abriendo un nuevo campo de intervención social capaz de poner a prueba la capacidad de la sociedad y sus instituciones para dar respuesta a las necesidades de las personas dependientes (mayores, enfermas y discapacitadas), permitiendo visibilizar, siquiera tímidamente, tales tareas de cuidado y propiciando una cobertura autónoma desde el punto de vista de la protección social, a través de la suscripción del oportuno convenio de Seguridad Social. El cuidado no profesional es una alternativa secundaria, no preferida ni prevalente en la opción legislativa, que acude a ella cuando no sea posible la atención a los dependientes mediante las prestaciones asistenciales o de servicios de la oferta pública o concertada de los servicios sociales. Sin embargo, quedan encuadrados (si bien voluntariamente tras el Real Decreto-ley 20/2012) en el sistema de Seguridad Social y se les incluye en el sistema de Formación Profesional para el empleo a través de acciones de formación y orientación, en parte para compensar el impacto negativo que su tarea de cuidados les puede originar en su carrera profesional o de seguro $^{21}$.

El tiempo transcurrido desde la implantación del SAAD permite ya poner al descubierto sus avances y logros, pero también sus principales déficits o carencias protectoras, fundamentalmente en cuanto hace al cuidado en el entorno familiar, al ser esta una materia impregnada por una indudable dimensión de género, necesitada, por tanto, de un profundo análisis desde tal perspectiva, prestando especial atención a la gestión de los cuidados en el entorno familiar, para comprobar si en el desarrollo de la Ley 39/2006 se tienen en cuenta las necesidades específicas de las mujeres cuidadoras (tanto profesionales como no profesionales) o, por el contrario, se están reforzando los clásicos roles que por cuestión de género se asignan en materia de cuidados ${ }^{22}$.

\footnotetext{
${ }^{21}$ SÁNCHEZ-URÁN AZAÑA. Y., "La protección de la dependencia", en AA.VV. (YZQUIERDO TOLSADA, M. y CUENA CASAS, M., Dirs.), Tratado de Derecho de Familia, Vol. III, Thomson/Aranzadi, Cizur Menor, 2007.

${ }^{22}$ A la vez que es importante que se consideren las necesidades específicas de las mujeres que reciben los cuidados, puesto que son mayoría entre la población de mayor edad con situación de dependencia reconocida. IMSERSO: Informe de la Comisión para el análisis de la situación actual del sistema de la dependencia, de la sostenibilidad y de los actuales mecanismos de financiación, para evaluar su adecuación a las necesidades asociadas a la dependencia, 6 de octubre de 2017, p. 103.
} 
Aunque no es posible profundizar en este momento en la materia, sí conviene recordar que la Ley 39/2006 regula diversos tipos de prestaciones económicas, entre las que figura la específicamente destinada a cuidados en el entorno familiar y apoyo a cuidadores no profesionales --compatible con algunas prestaciones económicas de Comunidades Autónomas ${ }^{23}--$.

La complejidad de la figura del cuidador informal viene dada por las diferentes situaciones en que se puede encontrar y, por ende, del diferente régimen jurídico en cada una de ellas. En principio la figura hace referencia a aquella persona (familiar o allegado, no profesional) que asiste al dependiente y le ayuda en la realización de las actividades básicas de la vida cotidiana. Por esa atención y cuidado, el ordenamiento laboral articula un conjunto de derechos destinados a facilitar la compatibilidad entre su prestación laboral y su responsabilidad en la atención a la dependencia. Ahora bien, a la hora de delimitar correctamente quién sea cuidador informal también debe tenerse en cuenta que la Ley 39/2006 dota al cuidador de medidas específicas de protección, de forma excepcional y en supuestos tasados en los términos previstos en los artículos 14.4 y $18^{24}$, teniendo en cuenta que la existencia del cuidado familiar es la que justifica la prestación económica, "que no parece que vaya a establecerse en razón de los rasgos (intensidad, tiempo) de la prestación familiar, sino del grado de dependencia del sujeto" 25 , estando además determinada en su cuantía por la capacidad económica del dependiente. La prestación económica no se establece como una renta de sustitución por haber renunciado a realizar una actividad profesional. Es más, el beneficiario de esta prestación económica es la persona en situación de dependencia y no el cuidador profesional, siendo la persona dependiente la que decide cómo utilizar la prestación, ya sea compensando económicamente a la persona cuidadora ya sea destinándola a otras finalidades ${ }^{26}$.

Esto es, una misma situación (atención al dependiente por cuidador no profesional) da lugar a la aplicación de diferentes regímenes jurídicos y las medidas de protección social van a ser distintas según se trate de un cuidador no formal que mantiene su relación laboral y la compagina con la labor de cuidado y atención al dependiente o de un cuidador

\footnotetext{
${ }^{23}$ Dando cuenta de todo el desarrollo autonómico, BLASCO LAHOZ, J.F., El sistema para la autonomía y la atención a la dependencia, Bomarzo, Albacete, 2009, pp. 26 y ss. o ALEGRE NUENO, M., "Régimen de incompatibilidades", en AA.VV. (VIQUEIRA PÉREZ, C. y ALEGRE NUENO, M., Coords.), Promoción de la autonomía personal y atención a las personas en situación de dependencia, Tirant lo Blanch, Valencia, 2013, pp. 79-80.

${ }^{24}$ Pese a que su bajo coste, una gestión administrativa relativamente rápida y barata, una oferta insuficiente de servicios y el respeto a la libertad de elección que tenía un peso creciente en el Programa Individual de Atención hicieran que la excepción se convirtiera "en la regla dominante". ERRANDONEA ULAZIA, E., "La protección social de los cuidadores familiares", en AA.VV. (AESSS), Protección a la familia y Seguridad Social: Hacia un nuevo modelo de protección sociolaboral, Laborum, Murcia, 2018, p. 154.

${ }^{25}$ GONZÁLEZ ORTEGA, S., "El cuidador no profesional de las personas en situación de dependencia en la Ley 39/2006", TL, núm. 89, 2007, p. 307.

${ }^{26}$ No faltan voces que cuestionan el destino de la misma, puesto que no se garantiza que la ayuda sea destinada al cuidador ni a los cuidados del dependiente. GARCÍA TESTAL, E., "Las medidas de conciliación de la vida laboral para la atención a la dependencia (Il): Las medidas de Seguridad Social y el convenio especial para los cuidados no profesionales", en AA.VV. (ROQUETA BUJ, R., Coord.), La situación de dependencia. Régimen jurídico aplicable tras el desarrollo reglamentario, estatal y autonómico de la Ley de Dependencia, Tirant lo Blanch, Valencia, 2009. pp. 791-792.
} 
informal que abandona su trabajo, temporal o definitivamente, o que no ha desarrollado actividad laboral alguna por dedicarse a cuidar al familiar o allegado (figura que, dicho sea de paso, encaja con el perfil de la cuidadora litigante en el proceso comentado, como potencial beneficiaria de pensión a favor de familiares, como compensación económica por dicha labor de cuidados procedente del sistema público de protección social).

La Ley 39/2006 define el cuidado no profesional como la atención prestada a personas en situación de dependencia en su domicilio, por personas "de su familia o de su entorno" no vinculadas a un servicio de atención profesionalizado. Evidentemente maneja el legislador una noción ambigua que ha sido acotada por la normativa reglamentaria de desarrollo y da cabida tanto al cónyuge y parientes "por consanguinidad, afinidad o adopción, hasta el tercer grado de parentesco" y a "las parejas de hecho, tutores y personas designadas, administrativa o judicialmente, con funciones de acogimiento" (artículo 12.2 Real Decreto 1051/2013) siempre que convivan en el mismo domicilio de la persona dependiente, esta esté siendo atendida por ellos y lo hayan hecho durante el período previo de un año a la fecha de presentación de la solicitud. Esto es, se amplía el concepto a personas que no sean familiares hasta el tercer grado, cuando la persona en situación de dependencia reconocida tenga su domicilio en un entorno caracterizado por insuficiencia de recursos públicos o privados acreditados, despoblación, o circunstancias geográficas o de otra naturaleza que impidan o dificulten otras modalidades de atención (incluida la atención mediante servicios a través de la prestación vinculada), en cuyo caso la Administración competente podrá excepcionalmente permitir la existencia de cuidados no profesionales por parte de una persona de su entorno que, aun no teniendo el grado de parentesco señalado ${ }^{27}$, resida en el municipio de la persona dependiente o en uno vecino, y lo haya hecho durante el periodo previo de un año a la fecha de presentación de la solicitud.

Desde el año 2007 se ha considerado oportuno delimitar el marco de encuadramiento en la Seguridad Social de los cuidadores profesionales. Con la aprobación del Real Decreto 615/2007 se integró a dicho colectivo en el Régimen General, en situación asimilada al alta a través de la suscripción de un convenio especial que además era financiado íntegramente por la Administración General del Estado, en concreto por el IMSERSO, a través de la firma del oportuno convenio con la TGSS ${ }^{28}$. Ni los cuidadores ni, por supuesto, las personas dependientes, habían de participar económicamente en dichas cotizaciones, confirmando la relación de cuidados que nace con el SAAD. La suscripción

\footnotetext{
${ }^{27}$ La ampliación del ámbito subjetivo de la titularidad de los derechos de conciliación no solo a casos de parentesco sino, incluso, a los supuestos en que no exista tal resulta más necesario, si cabe, al tener en cuenta que la mujer está abandonando progresivamente su rol tradicional de cuidadora del hogar familiar y se está incorporando plenamente al mercado de trabajo y que los vínculos familiares son cada vez más débiles. En propuesta de lege ferenda referida al derecho a la excedencia, SÁNCHEZ-RODAS NAVARRO, C., La excedencia por cuidado de familiares. El régimen jurídico de los cuidadores profesionales, Tirant lo Blanch, Valencia, 2008, p. 63 o, más recientemente, GIL PLANA, J., "La excedencia para el cuidado de hijos y familiares: puntos críticos", RMEYSS, núm. 133, 2017, p. 237.

${ }^{28}$ Para profundizar en las particularidades respecto al sujeto obligado y responsable de la cotización vid. FERNÁNDEZ ORRICO, F.J., "Prestación económica para cuidados en el entorno familiar y Seguridad Social de cuidadores no profesionales", RMTIM, núm. 74, 2008, pp. 97 y ss.
} 
del convenio quedaba supeditada al reconocimiento a la persona dependiente de la prestación económica de cuidados en el entorno familiar. Sin embargo, el elevado gasto que han supuesto dichas cotizaciones, al haberse convertido dicha prestación de cuidados en el entorno familiar en la protagonista, generando una deuda acumulada por dicho concepto, motivó la aprobación del Real Decreto-ley 20/2012, de 13 de julio, que vino a extinguir dichos convenios el 31 de agosto de 2012, manteniendo la posibilidad de su subsistencia, pero haciendo recaer la obligación de pago de dichas cotizaciones en el propio cuidador no profesional.

La justificación esgrimida sobre el conjunto de modificaciones adoptadas en el verano de 2012 ha sido muy plural, pero gira en torno a dos respuestas fundamentales. Por una parte, la insostenibilidad financiera del sistema y, por otra, la progresiva desvirtuación del modelo original, que defendía un sistema público de atención profesionalizada, invocándose finalmente las expectativas frustradas en la creación de empleo, que mejorarían significativamente si se desarrolla la red autonómica de servicios sociales. En apretada síntesis puede defenderse que con la reforma llevada a cabo en el año 2012 se han adoptado todo un conjunto de medidas en contra de la prestación de cuidados en el entorno familiar, hasta el punto de que un análisis integral de las mismas ha permitido a cierto sector doctrinal concluir con la idea de que dicha prestación ha quedado plenamente "estigmatizada" dentro del SAAD, pues se opta por un sistema que prefiere el reconocimiento de la prestación económica por asistencia personal, frente a la que se apoya en el cuidador familiar. Y lo que es más grave, la reforma de 2012 "relega de nuevo a estas mujeres que sacrifican su vida, su tiempo y su trabajo al cuidado de sus hijos, padres o cónyuge, al haberse reducido drásticamente las medidas de apoyo brindadas tradicionalmente por la LAAD, retornando al mundo de la invisibilidad en que han permanecido durante demasiado tiempo" 29 .

Las medidas de endurecimiento de los requisitos para el reconocimiento de la prestación para cuidado de familiar (básicamente reducción de su importe y exclusión del cuidador del sistema de Seguridad Social) provocaron una reducción progresiva y drástica del número de convenios especiales de cuidadores no profesionales, que han vuelto a revitalizarse en los dos últimos ejercicios. A fecha 31 de diciembre de 2020, el número total de convenios suscritos por los cuidadores no profesionales era de 60.293 , de los cuales, 6.615 fueron suscritos por hombres (un 11\%) y 53.678 por mujeres (el 89\% restante). Del total de convenios suscritos, un 38,8\% corresponde a menores de 50 años. En fin, los últimos datos publicados por el IMSERSO revelan que del conjunto de prestaciones del SAAD, un 31,57\% (en 2020) son prestaciones económicas para el cuidado de familiares, cifra esta que no permite considerarla como una prestación excepcional. No obstante, los datos expuestos son datos globales del conjunto del Estado y las diferencias son apreciables de una Comunidad Autónoma a otra, pero en síntesis

\footnotetext{
${ }^{29}$ MOLERO MARAÑÓN, $\mathrm{M}^{\mathrm{a}}$.L., "El reajuste o revisión profunda de las prestaciones económicas en el funcionamiento del SAAD: en especial, sobre la prestación por cuidados familiares", en AA.VV. (MOLERO MARAÑÓN, Ma.L., Coord.), Retos para una implantación efectiva del Sistema para la Autonomía y Atención a la Dependencia, Cinca, Madrid, 2013, p. 191.
} 
puede afirmarse que las prestaciones de servicios han aumentado de forma notoria y que, a pesar de la disminución apreciada en la prestación económica para cuidado de familiares, esta sigue manteniendo un peso importante dentro del sistema.

La prestación económica por cuidados en el entorno familiar tiene una doble finalidad. Por un lado, compensar el gasto que al dependiente le supone contar con el apoyo y cuidado del familiar o allegado que permanece en su entorno $\mathrm{y}$, por otro, resarcir económicamente a quien presta estas tareas. Como con acierto ha precisado la doctrina, la inexistente relación contractual entre el cuidador informal y la persona dependiente provoca que quien desempeña dicha tarea lo haga de manera "altruista por razón del vínculo de parentesco y afectivo" 30 con la persona en situación de dependencia, de tal modo que a día de hoy dichas personas (en su mayoría mujeres) continúan atendiendo a sus familiares con un soporte económico más bajo por los recortes en la prestación económica, retornando y emergiendo su labor de la trastienda de la invisibilidad, y con una tremenda frustración para las propias cuidadoras, al haberse generado unas expectativas que fueron cortadas de golpe ${ }^{31}$.

Como con acierto pronosticaron los primeros comentaristas de la reforma llevada a cabo en el año 2012, la nueva configuración de la prestación económica de cuidados en el entorno familiar había dejado de ser la prestación nuclear del sistema, acercándose a su concepción original que la define bajo el principio de "excepcionalidad" en su reconocimiento, pero lo más grave es que esta modificación (que afectaba a las posibilidades de acceder a los períodos mínimos de cotización exigidos por la ley) repercutió de forma desproporcionada sobre las mujeres y, como es conocido, no tardó en reflejarse en las estadísticas, pues de las 180.021 personas con convenio especial en julio de 2012 (de las que el 92,5\% eran mujeres) se pasó a 20.993 en marzo de 2013, y se descendió hasta las 8.000 en 2017, debido, fundamentalmente, a la falta de recursos de esas personas, mayoritariamente mujeres, se insiste, para asumir por su cuenta la cotización del convenio especial.

El 12 de julio de 2018, la Ministra de Sanidad, Consumo y Bienestar Social anunciaba que a finales de dicha anualidad, el Estado volvería a cotizar por las personas cuidadoras de familiares dependientes, lo cual, una vez materializado, ha permitido cambiar sustancialmente las expectativas de muchas personas cuidadoras. Como cabía esperar, dicha medida ha vuelto a dar un vuelco en la afiliación de estas personas en la Seguridad Social y en la suscripción del oportuno convenio especial por parte de las mismas. De acuerdo con las estimaciones del propio Gobierno, se esperaba que la medida beneficiara a unas 180.000 personas, derogando el recorte realizando en el año 2012, devolviendo a muchas de estas personas la posibilidad de causar pensiones de Seguridad Social por derecho propio y recuperando un importante elemento de seguridad de cara a su futuro.

\footnotetext{
${ }^{30}$ CRISTOBAL RONCERO, R., "Los cuidadores familiares y su visualización", en AA.VV. (AESSS), Protección a la familia y Seguridad Social: Hacia un nuevo modelo de protección sociolaboral, cit., p. 95. ${ }^{31}$ MOLERO MARAÑÓN, M ${ }^{a}$.L., Bases, evolución y retos de la Ley de Dependencia a los diez años de su aprobación, Bomarzo, Albacete, 2017, p. 88.
} 
Cumpliendo tal anuncio, y tras más de un lustro de recortes, el Real Decreto-ley 6/2019, de 1 de marzo, ha restablecido el abono de oficio de las cotizaciones al convenio especial de Seguridad Social que cubre a nuestros cuidadores no profesionales de dependientes, las cuales se abonan por el IMSERSO desde el 1 de abril de 2019, con la consiguiente protección "a efectos de las prestaciones de jubilación y de incapacidad permanente y muerte y supervivencia, derivadas de accidente, cualquiera que sea su carácter, o de enfermedad, con independencia de su naturaleza" 32 . Esta medida efectivamente ha permitido revertir los datos estadísticos, hasta el punto de que, a 31 de diciembre de 2020, la prestación económica para cuidados en el entorno familiar es recibida por 450.517 personas; es decir, "el 31,57\%" de las personas beneficiarias. En cualquier caso, dado que la suscripción de un convenio especial no garantiza que en todos los casos se vaya a generar una pensión pública de Seguridad Social (pues dada la variada casuística de situaciones entre las personas cuidadoras, algunas pueden estar más cerca de cumplir los requisitos de contributividad exigidos, pero en otras dicha posibilidad puede resultar remota) se presentan como realmente atractivas algunas iniciativas de entes públicos (Diputaciones provinciales ${ }^{33}$, por ejemplo), tendentes a la constitución de Entidades de Previsión Social voluntaria, que pueden resultar también de utilidad para personas que, por sus circunstancias, no han tenido la obligación de suscribir un convenio especial con la Seguridad Social, pero pueden tener interés en este instrumento de ahorro complementario de cara al futuro.

\section{Interpretación flexible del concepto de convivencia en las pensiones a favor de familiares}

Retomando la controversia jurídica objeto de comentario, conviene destacar que esta se cierne exclusivamente sobre la interpretación que debe darse al requisito de convivencia para obtener la pensión que se reclama. No se cuestiona el cumplimiento de los restantes requisitos (incluido el periodo mínimo temporal exigido de convivencia), por lo que el debate jurídico queda centrado en el concepto de convivencia legalmente exigido, habiendo entendido el INSS que no se cumple porque la demandante y la causante se hallaban empadronadas en pisos diferentes, a pesar de convivir en el mismo edificio. Y estando en juego una prestación que en la práctica viene siendo percibida casi en un $70 \%$ por mujeres, considera la Sala de lo Social del Tribunal Superior de Justicia de Canarias que el requisito de convivencia debe interpretarse de forma flexible y abierta, y no

\footnotetext{
${ }^{32}$ Cfr. artículo 3 Real Decreto 615/2007. Sobre la situación anterior a esta enmienda legal, con cita de estadísticas alarmantes acerca de la caída en picado de las cifras de convenios especiales suscritos por cuidadores no profesionales, vid. MARTÍNEZ GIRÓN, J., "La sostenibilidad del modelo español actual de protección social por dependencia. Un estudio crítico-comparativo con la sostenibilidad del modelo alemán”, $R D S$, núm. 72, 2015, pp. 63-64.

${ }^{33}$ A título de ejemplo vid. ERRANDONEA ULAZIA, E., "La protección social de los cuidadores familiares", en AA.VV. (AESSS), Protección a la familia y Seguridad Social: Hacia un nuevo modelo de protección sociolaboral, cit., pp. 153 y ss.
} 
mecánica y formalista, pues de ser así se quedarían fuera de protección situaciones reales de necesidad y dependencia, padecidas mayoritariamente por las mujeres.

Para llegar a tal conclusión la Sala hace un pormenorizado repaso de los diversos criterios hermenéuticos.

Desde un punto de vista estrictamente literal, se considera que el requisito de convivencia "aparece desnudo sin condicionarse a un domicilio único o a un empadronamiento común", pues según el diccionario normativo de la RAE, el verbo "convivir" se describe como "vivir en compañía de otro u otros" y a su vez, la primera acepción de "vivir" se define como "tener vida".

En un contexto sistemático, la Sala interpreta dicho concepto de acuerdo con la sociedad actual "teniendo en cuenta las necesidades y los valores familiares de la sociedad de este siglo, donde la intimidad familiar y personal adquieren un valor superior al de otras épocas, al tener una protección supralegal, como derecho fundamental reconocido en la Constitución (artículo 18.1 CE). Tal intimidad puede traducirse en mantener la independencia de una vivienda propia donde no por ello se excluye el cuidado, el socorro y la asistencia diaria por parte de otros familiares".

Por último, desde un punto de vista teleológico o finalista, se recuerda que la finalidad de las pensiones a favor de familiares es dar la adecuada cobertura a una "situación de cualificada necesidad en razón a la pérdida del causante de la prestación, cuyos ingresos venían a constituir el sustento de la familia" ${ }^{34}$. Esa finalidad asistencial de las prestaciones debatidas ha llevado a la jurisprudencia ${ }^{35}$ a matizar la interpretación del requisito de convivencia, superando la literalidad de la expresión y el concepto formal de convivencia, pues lo que debe valorarse no es tanto la cohabitación sino la práctica de los cuidados respecto del familiar necesitado de ello y la satisfacción de las necesidades físicas, psíquicas, morales y espirituales de la persona impedida. Por tanto, debe interpretarse la convivencia, no en la literalidad de sus términos, sino con un criterio más amplio a raíz de supuestos excepcionales impuestos por circunstancias transitorias de trabajo fuera de la residencia habitual con la finalidad de atender mejor al sostenimiento de la familia cuando las relaciones afectivas y económicas no han desaparecido.

Dicho criterio de interpretación flexible y humanizador del concepto de convivencia, mantenido por el Tribunal Supremo, que prioriza la efectividad de las atenciones y

\footnotetext{
${ }^{34}$ STS de 24 de febrero de 1995 (Rec. 2790/ 1994) o STC 3/1993, de 14 de enero (RTC 1993/ 3), poniendo de relieve dicha finalidad en los siguientes términos: "Es claro que su finalidad última es remediar la situación de necesidad (...), la norma atiende en todos los casos a auténticas situaciones de necesidad, dada la incompatibilidad de esta pensión con la percepción de ingresos propios por parte de los beneficiarios. Su función debe ser considerada como el otorgamiento de rentas de subsistencia a quienes, por carecer de todo tipo de ingresos, se encuentran en un estado de necesidad (...)".

${ }^{35}$ STS de 9 de febrero de 1985 (Rec. 355/1984).
} 
cuidados y no tanto el formalismo de la cohabitación física ha sido seguido (como recuerda la Sala Social del Tribunal Superior de Justicia de Canarias) por otras Salas ${ }^{36}$.

\section{Interpretación del requisito de convivencia con perspectiva de género}

Una vez detectado el impacto de género que tiene la prestación, considera la Sala que es obligación de todo órgano jurisdiccional integrar la perspectiva de género en la impartición de justicia, que debe implementarse como metodología de resolución en toda controversia judicial en la que se involucren relaciones asimétricas o patrones estereotípicos de género, aun cuando las partes no lo soliciten expresamente, a tenor de lo previsto en los artículos $1,10.2^{\circ}, 9.2^{\circ}, 14$ y $96 \mathrm{CE}$; artículos $5.1^{\circ}, 7.1^{\circ}$ y $2^{\circ} \mathrm{LOPJ}$, en relación con los artículos 4 y 15 LOIEMH y los artículos $2 . c$ ), d) e) y $11.1^{\circ}$ de la Convención para la eliminación de todas las formas de discriminación contra la Mujer (CEDAW), que exigen de los poderes públicos del Estado la "debida diligencia" en la aplicación de los estándares y obligaciones internacionales en materia de género.

El principio de integración de la dimensión de género en la actividad jurídica vincula a todos los Poderes del Estado (legislativo, ejecutivo y judicial) y, en concreto, la vinculación de la actividad jurisdiccional del Poder Judicial (dada su independencia) deriva de su sumisión al imperio de la ley (ex artículo 117 CE). Tal afirmación enlaza necesariamente con la existencia de un amplio derecho antidiscriminatorio, con amparo constitucional en el artículo 14, en conexión con el 9.2 y se halla normativizado en los artículos 4 y 15 LOIEMH, que según el pronunciamiento comentado debe desplegarse en tres fases judiciales concretas:

$1^{\circ}$.- En la tramitación del procedimiento, mediante cláusulas de protección jurisdiccional, flexibilizadoras del rigor procesal para garantizar la tutela de las víctimas.

$2^{\circ}$.- En la valoración de la prueba, a través de la distribución de la carga de la prueba de la discriminación y relevancia de la declaración de la víctima.

$3^{\circ}$.- En la aplicación e interpretación de las normas sustantivas, a través de la prohibición de la discriminación directa e indirecta, medidas de acción positiva, democracia paritaria e igualdad de oportunidades, derechos de maternidad y conciliación o protección frente a la violencia de género.

\footnotetext{
${ }^{36}$ Entre otras, SSTSJ de Andalucía/Sevilla de 7 de septiembre de 2016 (Rec. 2131/2015); País Vasco de 22 de noviembre de 2016 (Rec. 2174/2016) o Galicia de 27 de enero de 2012 (Rec. 3546/ 2008). Esta última aproxima las prestaciones a favor de familiares a un concepto asistencial de la protección, recordando la doctrina del extinto Tribunal Central de Trabajo --SSTCT de 29 febrero 1988 (RTCT 1988, 93), 14 de julio 1987 y 17 mayo 1983-- que vino considerando que el requisito de convivencia no podía exigirse en un sentido literal y estricto de cohabitación física y material; "pues más que a la materialidad de la permanencia bajo el mismo techo, ha de atenderse al nexo de ayuda, socorro, colaboración material y espiritual, o dicho de otra forma, en el sentido de permanencia de la relación personal, directa y frecuente".
} 
A partir de tales premisas, juzgar con perspectiva de género debe ser una labor judicial que conlleve la utilización de criterios de sustitución o de comparación hipotética para verificar si, en una situación dada, un hombre habría sido tratado de la misma manera en que lo ha sido una mujer; la consideración de la situación de marginalidad real o potencial, o de victimización secundaria, en la cual se puede encontrar la mujer a la hora de valorar su conducta y la integración del valor de igualdad de los sexos en la aplicación de una norma que, debiendo considerarlo, no ha considerado ese valor, o laguna axiológica, evitando determinados efectos perversos.

Por tanto, considera la Sala que la interpretación del Derecho con perspectiva de género exige "la contextualización y la actuación conforme al principio pro persona, que se configura en este ámbito como un criterio hermenéutico que obliga a los órganos jurisdiccionales a adoptar interpretaciones jurídicas que garanticen la mayor protección de los derechos humanos".

La aplicación de esta hermenéutica interpretativa a la pensión a favor de familiares, "tratándose de una prestación que viene siendo percibida casi en un $70 \%$ por mujeres, exige interpretar el requisito de convivencia de forma flexible, abierta y no mecánica y formalista, pues ello puede excluir del acceso a las prestaciones situaciones de necesidad y dependencia padecida mayoritariamente por las mujeres".

Aplicando dicho canon hermenéutico al caso comentado, se comprueba que la demandante había venido cuidando y atendiendo las necesidades vitales de su madre mientras vivía y lo requería (pues, tal y como se recoge en la fundamentación jurídica de la sentencia, ello no se cuestiona ni se discute). Por tanto, "los cuidados empleados en la madre, su asistencia continua, el estar cercana de la misma durante el día y la noche al vivir en el mismo edificio, debe priorizarse en la valoración de este requisito, que tiene un componente claramente social, humano y asistencial, que quedaría diluido en una interpretación mecanicista y puramente física".

En concreto, el hecho de vivir en plantas diferentes $\left(1^{\mathrm{a}}\right.$ y $\left.2^{\mathrm{a}}\right)$ del mismo edificio, madre e hija, "no puede servir para que, en base a criterios rigoristas, se niegue el acceso de la hija cuidadora, a la pensión solicitada", pues tal y como había quedado probado, "la actora convivía en el piso superior con su hijo de 19 años de edad, que también ha requerido de cuidados y asistencia durante su crianza por parte de la hija de la causante, que también es madre de su nieto. No entender la realidad del funcionamiento de los cuidados de familiares (ascendientes, descendientes, etc.) y devaluarlos hasta el extremo de opacarlos tras la exigencia formalista de la cohabitación física en la misma planta de un edificio común, es vaciar de contenido la finalidad misma de la prestación a favor de familiares (cuidadores), lo que tiene sin duda un impacto estadístico mayor en el sexo femenino, pudiéndose incurrir, en este caso, en una discriminación indirecta por razón de género". 
En fin, para apuntalar su argumentación, recuerda la Sala la doctrina sentada en la sentencia del Tribunal Supremo de 29 de enero de $2020^{37}$ que no hace sino modificar su criterio jurisprudencial en relación al acceso de las prestaciones a favor de familiares derivadas de pensionista SOVI, aplicando la perspectiva de género en la interpretación del derecho.

Se recuerda, asimismo, la obligación judicial de integrar esta hermenéutica acorde con los mandatos internacionales, en tanto resulta indubitada la obligación de jueces y tribunales de incorporar la perspectiva de género en lo que constituye su actuación como Poder del Estado, esto es, en la interpretación y aplicación de las normas ${ }^{38}$.

Por ello, la interpretación estricta y literal del artículo 226 TRLGSS en el caso comentado "puede generar un impacto de género, una discriminación indirecta, al desplegar efectos desproporcionados sobre el colectivo femenino", y como bien recuerda la Sala, el principio de igualdad de trato exige eliminar, no sólo la discriminación abierta, sino también toda forma de discriminación encubierta que, por la aplicación de otros criterios, lleve, de hecho, al mismo resultado.

\section{Conclusión final}

A pesar de que las reformas institucionales introducidas en el año 2012 en el contexto de la Ley de Dependencia han pretendido fomentar las prestaciones de servicios (mayor calidad, generación de empleo, retorno económico) y reducir el déficit público, lo cierto es que las personas cuidadoras no profesionales (fundamentalmente mujeres) siguen realizando una tarea imprescindible para la sociedad que no es posible desconocer. Por tanto, si se minora el gasto en el cuidado no profesional a sabiendas de que se realizará sin ese coste económico y sin valorar "el coste social", en realidad lo que se está produciendo es un retroceso claro en el Estado social y en la igualdad entre hombres y mujeres ${ }^{39}$.

La escasa libertad de elección de los usuarios a la hora de cubrir sus necesidades lleva consigo un fuerte impacto negativo de género que pone en evidencia dos datos constatables: primero, que muchas mujeres se dedican a estos cuidados no por libre elección, sino por imposición económica y social (y sin cobertura ni consideración) y,

\footnotetext{
${ }^{37}$ Rec. 3097/2017. Enfoque de género que pretende compensar la discriminación que sufre la mujer por su situación en el mercado de trabajo y la proyección de la misma en el ámbito de la protección social, atribuyendo al Alto Tribunal la función de adaptación normativa a la realidad vigente.

${ }^{38}$ Sostenido también en las SSTS de 26 de septiembre de 2018 (Rec. 1352/2017) y 13 de noviembre de 2019 (Rec. 75/2018).

${ }^{39}$ FERNÁNDEZ COLLADOS, M‥B., "Los cuidados en el entorno familiar más de una década después: los vaivenes en su regulación, su aplicación por la doctrina judicial y sus efectos en materia de igualdad", REDT, núm. 210, 2018, p. 12.
} 
segundo, que los hombres no asumen en igualdad el papel de cuidadores, con lo que contribuyen a aumentar un reparto discriminatorio de las labores de cuidado ${ }^{40}$.

Como es fácil adivinar, la situación descrita de un mayor protagonismo femenino en tareas de cuidados familiares, tiene una indudable repercusión en el acceso a prestaciones de Seguridad Social, pese a que las normas reguladoras de tal protección sean neutras en cuanto al género. No hay que olvidar que el sistema público fue diseñado bajo un modelo social en el cual las funciones familiares y laborales de hombres y mujeres respondían a patrones culturales significativamente distintos de los actuales y de ahí la oportunidad de realizar una interpretación normativa con perspectiva de género, pues como con acierto ha señalado el propio Consejo General del Poder Judicial, interpretar con perspectiva de género exige "razonar con una lógica distinta de las épocas pasadas y que resulte útil para remover los obstáculos que dificultan la igualdad efectiva" ${ }^{\text {"41. }}$.

La Sala Cuarta del Tribunal Supremo ha incorporado el método jurídico de juzgar con perspectiva de género de forma puntual desde hace más de diez años ${ }^{42} \mathrm{y}$, aunque en la actualidad sigue sin estar integrado de forma plena, se puede apreciar una tendencia a su aplicación rigurosa de manera cada vez más sólida en los pronunciamientos recientes de la Sala de lo Social. De hecho, el rigor en la aplicación de esta metodología de enjuiciamiento no se compadece con su uso como modismo metodológico o cláusula de estilo de aplicación automática en todos los casos, sino que debe llevar a los tribunales a discernir cuándo su incorporación en el razonamiento jurídico es pertinente como criterio autónomo y operativo.

Es más, prescindir del análisis con perspectiva de género en el proceso de enjuiciamiento por considerarlo una mera opción hermenéutica del órgano judicial puede tener implicaciones graves que se reflejan en la pérdida de derechos laborales y sociales de las mujeres, de ahí que se considere plenamente acertada la aplicación del canon de equidad de género en el asunto comentado, a la vez que se comparte la urgencia y necesidad de ratificación del Convenio núm. 190 de la OIT, que permitiría sancionar la actuación empresarial de violencia laboral contra la mujer por su rol de cuidadora.

En fin, la sentencia comentada es un ejemplo más de cómo la perspectiva de género está siendo integrada de forma progresiva en todos los niveles jurisdiccionales y ello no solo por incorporar esta metodología de enjuiciamiento cada vez en mayor número de asuntos, sino por hacerlo de modo riguroso y sin recurrir a su invocación como mero hábito o cláusula de estilo.

En la mayoría de los casos, los Tribunales Superiores de Justicia y el propio Tribunal Supremo hacen un uso adecuado y necesario de dicha metodología jurídica en sus

\footnotetext{
40 ASOCIACIÓN ESTATAL DE DIRECTORES Y GERENTES EN SERVICIOS SOCIALES: XVIII Dictamen del Observatorio de la Ley 39/2006 de Promoción de la Autonomía Personal y Atención a las Personas en situación de Dependencia, 31 de diciembre de 2017, pp. 23 y 24.

${ }^{41}$ CGPJ: Guía Práctica de la LO 1/2004, de 28 de diciembre, de Medidas de Protección Integral contra la Violencia de Género, 2016, p. 288.

${ }^{42}$ Desde la STS de 21 de diciembre de 2009 (Rec. 201/2009).
} 
pronunciamientos, consiguiendo con ello importantes avances en materia de igualdad efectiva, al detectar y corregir prácticas discriminatorias. Sin embargo, siguen detectándose supuestos en los que los Tribunales ceden a la formalidad y, prescindiendo del análisis con enfoque de género, se mantienen apegados a la interpretación formal y literal estricta. Se trata de casos en los que no se llega a descender a la valoración del fondo del asunto, sino que se aplican normas procedimentales o procesales obviando por completo la situación fáctica o contexto de la situación controvertida ${ }^{43}$.

Se precisa, por tanto, seguir avanzando para que el compromiso de los Tribunales laborales con el enfoque de género se extienda también a la aplicación e interpretación de las normas procesales (como la valoración de la contradicción ex artículo 219 LRJS) admitiendo su aplicación flexible o modulada cuando ello sea necesario por apreciarse que la rigidez de tales normas es el obstáculo que dificulta la igualdad efectiva (artículo $9.2 \mathrm{CE})^{44}$.

En el caso objeto de comentario se recuerda que el propio Tribunal Supremo, desde antiguo, viene matizando el concepto de convivencia exigido para acceder a las prestaciones en favor de familiares, interpretando dicho requisito, no en la literalidad de la palabra, sino con un criterio más amplio en razón a supuestos excepcionales impuestos por las particulares circunstancias, y estas particulares circunstancias son incuestionables en el caso analizado, pues no se ha negado que la recurrente efectivamente cuidara de su madre "de forma continuada" aunque no convivieran juntas. Es cierto que no convivían en el mismo domicilio, pero sí en el mismo edificio, en plantas diferentes y, por ello, no puede negarse a la hija cuidadora la pensión solicitada, pues no cabe devaluar los cuidados familiares hasta el extremo de "opacarlos" o "invisibilizarlos" bajo una formalista exigencia de cohabitación física en el mismo domicilio.

\section{Bibliografía}

ALEGRE NUENO, M., "Régimen de incompatibilidades", en AA.VV. (VIQUEIRA PÉREZ, C. y ALEGRE NUENO, M., Coords.), Promoción de la autonomía personal y atención a las personas en situación de dependencia, Tirant lo Blanch, Valencia, 2013.

AVILÉS PALACIOS, L., "Juzgar con perspectiva de género. Por qué y para qué”, AMJE (Asociación de Mujeres Juezas de España), 29 de agosto de 2017.

\footnotetext{
${ }^{43}$ Ello sucede, por ejemplo, con la doctrina legal surgida a propósito del enjuiciamiento de las políticas de externalización empresarial generalizadas en las cadenas hoteleras respecto a departamentos feminizados (camareras de pisos) que, al ser analizadas omitiendo el canon de equidad de género, acaban confirmando la precarización de las condiciones laborales de las personas trabajadoras afectadas, especialmente en materia retributiva.

${ }^{44}$ RODRÍGUEZ GONZÁLEZ, S., "La perspectiva de género en la aplicación e interpretación de las normas por la Sala de lo Social del Tribunal Supremo", op. cit., p. 16.
} 
BLASCO LAHOZ, J.F., El sistema para la autonomía y la atención a la dependencia, Bomarzo, Albacete, 2009.

CGPJ: Guía Práctica de la LO 1/2004, de 28 de diciembre, de Medidas de Protección Integral contra la Violencia de Género, 2016.

CRISTOBAL RONCERO, R., "Los cuidadores familiares y su visualización", en AA.VV. (AESSS), Protección a la familia y Seguridad Social: Hacia un nuevo modelo de protección sociolaboral, Laborum, Murcia, 2018.

ERRANDONEA ULAZIA, E., "La protección social de los cuidadores familiares", en AA.VV. (AESSS), Protección a la familia y Seguridad Social: Hacia un nuevo modelo de protección sociolaboral, Laborum, Murcia, 2018.

FERNÁNDEZ COLLADOS, $M^{\mathrm{a}}$.B., "Los cuidados en el entorno familiar más de una década después: los vaivenes en su regulación, su aplicación por la doctrina judicial y sus efectos en materia de igualdad", REDT, núm. 210, 2018.

FERNÁNDEZ ORRICO, F.J., "Prestación económica para cuidados en el entorno familiar y Seguridad Social de cuidadores no profesionales”, RMTIM, núm. 74, 2008.

GARCÍA TESTAL, E., "Las medidas de conciliación de la vida laboral para la atención a la dependencia (Il): Las medidas de Seguridad Social y el convenio especial para los cuidados no profesionales", en AA.VV. (ROQUETA BUJ, R., Coord.), La situación de dependencia. Régimen jurídico aplicable tras el desarrollo reglamentario, estatal y autonómico de la Ley de Dependencia, Tirant lo Blanch, Valencia, 2009.

GIL PLANA, J., "La excedencia para el cuidado de hijos y familiares: puntos críticos", RMEYSS, núm. 133, 2017.

GONZÁLEZ ORTEGA, S., "El cuidador no profesional de las personas en situación de dependencia en la Ley 39/2006”, TL, núm. 89, 2007.

JIMÉNEZ HIDALGO, A., "Juzgar con perspectiva de género en la jurisdicción de lo Social. ¿Es necesaria una reforma legislativa?”, Revista de la Comisión de lo Social de Juezas y Jueces para la Democracia, núm. 197, 2019.

LOUSADA AROCHENA, J.F., El derecho fundamental a la igualdad efectiva de mujeres y hombres, Tirant lo Blanch, Valencia, 2014.

LOUSADA AROCHENA, J.F., "La integración de la perspectiva de género en la aplicación e interpretación de las normas por la jurisdicción social”, RDS, núm. 76, 2016.

MARTÍNEZ BARROSO, Ma ${ }^{a}$.R., "La protección social de las trabajadoras: la brecha de género en las prestaciones de jubilación y desempleo", en AA.VV. (RODRÍGUEZ ESCANCIANO, S. y MARTÍNEZ BARROSO, Ma.R., Dirs. y ÁLVAREZ CUESTA, H., Coord.), La empleabilidad y la calidad en el empleo: apostando por la igualdad efectiva, Sepín, Madrid, 2019. 
MARTÍNEZ GIRÓN, J., "La sostenibilidad del modelo español actual de protección social por dependencia. Un estudio crítico-comparativo con la sostenibilidad del modelo alemán”, RDS, núm. 72, 2015.

MOLERO MARAÑÓN, Ma.L., "El reajuste o revisión profunda de las prestaciones económicas en el funcionamiento del SAAD: en especial, sobre la prestación por cuidados familiares", en AA.VV. (MOLERO MARAÑÓN, Mª.L., Coord.), Retos para una implantación efectiva del Sistema para la Autonomía y Atención a la Dependencia, Cinca, Madrid, 2013.

MOLERO MARAÑÓN, Ma.L., Bases, evolución y retos de la Ley de Dependencia a los diez años de su aprobación, Bomarzo, Albacete, 2017.

MOLINA NAVARRETE, C., La doctrina jurisprudencial por discriminación de género en el orden social, Wolters kluwer, Madrid, 2020.

POYATOS I MATAS, G., “Juzgar con perspectiva de género: una metodología vinculante de justicia equitativa", IQual. Revista de Género e Igualdad, núm. 2, 2019.

POYATOS I MATAS, G., "Nueva sentencia con enfoque de género en el ejercicio del derecho a cuidar y trabajar: A propósito de la Sentencia de la Sala de lo Social del TSJ de Canarias de 27 agosto 2019 (rec. 533/2019)", IQual. Revista de Género e Igualdad, núm. 3, 2020.

RIVAS VALLEJO, P., "Las beneficiarias de pensiones SOVI pueden causar prestaciones en favor de familiares", Revista de Jurisprudencia Laboral, núm. 2, 2020.

RODRÍGUEZ GONZÁLEZ, S., "La perspectiva de género en la aplicación e interpretación de las normas por la Sala de lo Social del Tribunal Supremo", Trabajo y Derecho, núm. 76, 2021.

SÁNCHEZ-RODAS NAVARRO, C., La excedencia por cuidado de familiares. El régimen jurídico de los cuidadores profesionales, Tirant lo Blanch, Valencia, 2008.

SÁNCHEZ-URÁN AZAÑA. Y., "La protección de la dependencia", en AA.VV. (YZQUIERDO TOLSADA, M. y CUENA CASAS, M., Dirs.), Tratado de Derecho de Familia, Vol. III, Thomson/Aranzadi, Cizur Menor, 2007.

VILLAR CAÑADA, I.M., "La necesaria interpretación del sistema de Seguridad Social desde un enfoque de género. Comentario a la Sentencia del Tribunal Supremo (Sala de lo Social) núm. 79, de 29 de enero", Revista de Derecho de la Seguridad Social, núm. 26, 2021.

\section{Anexo}

ASOCIACIÓN ESTATAL DE DIRECTORES Y GERENTES EN SERVICIOS SOCIALES: XVIII Dictamen del Observatorio de la Ley 39/2006 de Promoción de la 
Autonomía Personal y Atención a las Personas en situación de Dependencia, 31 de diciembre de 2017.

IMSERSO: Informe de la Comisión para el análisis de la situación actual del sistema de la dependencia, de la sostenibilidad y de los actuales mecanismos de financiación, para evaluar su adecuación a las necesidades asociadas a la dependencia, 6 de octubre de 2017. 